Published in final edited form as:

Neuroimage. 2013 January 1; 64: . doi:10.1016/j.neuroimage.2012.09.021.

\title{
Associations Between White Matter Microstructure and Infants' Working Memory
}

\author{
Sarah J. Shortt ${ }^{a, b}$, Jed T. Elison ${ }^{b, c, 1}$, Barbara Davis Goldman ${ }^{c, d}$, Martin Styner ${ }^{a, e}$, Hongbin \\ Gu $^{a, f}$, Mark Connelly ${ }^{g}$, Eric Maltbie ${ }^{a}$, Sandra Woolson ${ }^{a}$, Weili Lin ${ }^{h, i}$, Guido Gerig', J. Steven \\ Reznick $^{\mathrm{c}, \mathrm{d}}$, and John H. Gilmore ${ }^{\mathrm{a}, \mathrm{i}}$ \\ aDepartment of Psychiatry, University of North Carolina, Chapel Hill, NC \\ ${ }^{b}$ Carolina Institute for Developmental Disabilities, University of North Carolina, Chapel Hill, NC \\ 'Department of Psychology, University of North Carolina, Chapel Hill, NC \\ ${ }^{d}$ Frank Porter Graham Child Development Institute, University of North Carolina, Chapel Hill, NC \\ eDepartment of Computer Science, University of North Carolina, Chapel Hill, NC \\ fDepartment of Biostatistics, University of North Carolina, Chapel Hill, NC \\ gDepartment of Pediatrics, University of North Carolina, Chapel Hill, NC \\ hDepartment of Radiology, University of North Carolina, Chapel Hill, NC \\ 'Biomedical Research Imaging Center, University of North Carolina, Chapel Hill, NC \\ iScientific Computing and Imaging Institute, University of Utah, Salt Lake City, UT
}

\section{Abstract}

\begin{abstract}
Working memory emerges in infancy and plays a privileged role in subsequent adaptive cognitive development. The neural networks important for the development of working memory during infancy remain unknown. We used diffusion tensor imaging (DTI) and deterministic fiber tracking to characterize the microstructure of white matter fiber bundles hypothesized to support working memory in 12-month-old infants $(n=73)$. Here we show robust associations between infants' visuospatial working memory performance and microstructural characteristics of widespread white matter. Significant associations were found for white matter tracts that connect brain regions known to support working memory in older children and adults (genu, anterior and superior thalamic radiations, anterior cingulum, arcuate fasciculus, and the temporal-parietal segment). Better working memory scores were associated with higher FA and lower RD values in these selected white matter tracts. These tract-specific brain-behavior relationships accounted for a significant amount of individual variation above and beyond infants' gestational age and
\end{abstract}

(C) 2012 Elsevier Inc. All rights reserved.

Corresponding Author Sarah J Short, PhD Department of Psychiatry UNC School of Medicine Med Wing C, Rm 365 Chapel Hill, NC, 27599 608-770-1357 (phone) 919-966-7659 (fax) sjshort@med.unc.edu.

${ }^{1}$ Department of Humanities and Social Sciences, California Institute of Technology, Pasadena, CA

Parts of this work were presented at the biennial meeting of the Society for Research in Child Development, Montreal, Canada, April 2011.

Disclosure Statement The authors have no actual or potential conflict of interest to report including any financial, personal or other relationships with other people or organizations within three years of beginning the work submitted that could inappropriately influence the results reported herein.

Publisher's Disclaimer: This is a PDF file of an unedited manuscript that has been accepted for publication. As a service to our customers we are providing this early version of the manuscript. The manuscript will undergo copyediting, typesetting, and review of the resulting proof before it is published in its final citable form. Please note that during the production process errors may be discovered which could affect the content, and all legal disclaimers that apply to the journal pertain. 
developmental level, as measured with the Mullen Scales of Early Learning. Working memory was not associated with global measures of brain volume, as expected, and few associations were found between working memory and control white matter tracts. To our knowledge, this study is among the first demonstrations of brain-behavior associations in infants using quantitative tractography. The ability to characterize subtle individual differences in infant brain development associated with complex cognitive functions holds promise for improving our understanding of normative development, biomarkers of risk, experience-dependent learning and neuro-cognitive periods of developmental plasticity.

\section{Keywords}

infant; working memory; white matter; diffusion tensor imaging; brain development

\section{Introduction}

Working memory is an early, emerging cognitive building block that plays a formative role in the development of other executive functions early in life (Diamond, 1985, 1991; Zelazo et al., 2008). Broadly speaking, working memory is the capacity to temporarily maintain and subsequently manipulate information in the service of goal-oriented actions (Baddeley, 1992). Visuospatial working memory emerges around six months of age, which is followed by a dramatic increase in memory capacity that continues to improve into early adulthood (Diamond et al., 2005; Pelphrey et al., 2004; Reznick et al., 2004; Zald and Iacono, 1998; Zelazo et al., 1996). Individual differences in working memory performance are associated with language development, problem solving, and complex reasoning (Reznick, 2009), but the sources of these differences remain unknown. In school-aged children and adults, performance on working memory tasks is supported by a clearly delineated set of neural circuits that appears to refine from late childhood to adulthood as the function of brain networks become more specialized (Durston et al., 2006; Fair et al., 2007; Klingberg, 2006; Nagy et al., 2004; Scherf et al., 2006; Uddin et al., 2011). Evidence from positron emission tomography, functional magnetic resonance imaging, and diffusion tensor imaging (DTI) has identified a set of overlapping brain regions--prefrontal, frontal, temporal, and parietal regions--that support working memory processes during childhood and adulthood (Courtney et al., 1997; Jonides et al., 1993; Klingberg et al., 2002, 2006; Kwon et al., 2002; Nagy et al., 2004). The neural networks and brain regions that support working memory during infancy, however, have yet to be determined. Thus, we sought to investigate the association between short-term visuospatial working memory performance and the putative neural circuits underlying this cognitive capacity in 12-month-old infants.

Efficient signal transmission allows for efficient information processing. This relationship depends in part on the organizational integrity of white matter connections within the brain. Animal and postmortem studies have shown that the most prolific period of white matter myelination occurs between mid-gestation and two years of age (Brody et al., 1987; Yakovlev and LeCours, 1967). Until recently, technological and methodological limitations have prevented researchers from non-invasively characterizing this critical period of dynamic maturation. Diffusion tensor imaging (DTI) has emerged, however, as a way to non-invasively measure white matter microstructure in vivo throughout the life span and has been used to investigate changes in white matter at different stages of brain development, including during infancy (Dubois et al., 2009; Gao et al., 2009a; Geng et al., 2012a; Lebel et al., 2008; Mukherjee et al., 2001; Paus et al., 2001; Schmithorst et al., 2002).

Diffusion measures commonly used to characterize microstructural features of white matter include fractional anisotropy (FA), and the apparent diffusion coefficients axial diffusivity 
(AD) and radial diffusivity (RD). While FA has been more widely used to describe diffusion anisotropy in brain tissue, Song et al., $(2003 ; 2005)$ suggested that greater insight about the underlying microstructural properties can be derived from separating the three eigenvalues that comprise FA to reflect diffusion parallel to $\left(\mathrm{AD}=\lambda_{1}\right)$ and perpendicular to $\left(\mathrm{RD}=\left(\lambda_{2}+\right.\right.$ $\left.\left.\lambda_{3}\right) / 2\right)$ ), the sampled white matter fibers. Using these additional indices of diffusion to better characterize white matter microstructure, recent tract-based longitudinal DTI studies of infant brain development (Gao et al., 2009a; Geng et al., 2012a; 2012b) showed dramatic changes in FA, RD, and $\mathrm{AD}$ during the first two years of life. The developmental trajectories of these diffusion properties shows tract-specific heterogeneity with more rapid changes over the first year of life, compared to the second. Analysis of neural network formation in infancy also reveals rapid changes in the development of functional neural networks that resemble adult-like default networks by two years of age (Gao et al., 2009b, 2011). Research efforts to non-invasively characterize infant brain development and its functional correlates represent a burgeoning field in developmental cognitive neuroscience, with great promise and many questions to address.

Microstructural characteristics of white matter fiber bundles have also been described in relation to diseases (multiple sclerosis, schizophrenia), developmental disorders (cerebral palsy, autism), and cognitive capacities (language, working memory) (Karlsgodt et al., 2008; Schmithorst et al., 2005; Thomas et al., 2005; Werring et al., 1999; Wolff et al., 2012). However, the majority of studies examining associations between white matter and cognitive ability have been conducted in older children, adolescents, and aging populations. These investigations have reported differences in white matter characteristics associated with information processing speed, cognitive control, language, reading/literacy, and arithmetic competence, among other skills (Bengtsson et al., 2005; Catani et al., 2007; Hu et al., 2011; Liston et al., 2006; Nagy et al., 2004; Penke et al., 2010). Thus, there is a need to study how and when the structural characteristics of white matter influence the formation of cognitive functions. Identifying the structural characteristics associated with individual differences in working memory early in life could provide an early indicator of impending deficits, as well as clues for how to foster optimal learning (Keller and Just, 2009; Markham and Greenough, 2004; Quartz and Sejnowski, 1997). Furthermore, deficits in working memory are a core feature of many developmental and neuropsychiatric disorders (Gottesman and Gould, 2003). Accurately identifying early brain-behavior relationships could help us better understand such disorders and prevent downstream effects that poor working memory can have for competency in other cognitive domains, including language, reading, planning, and mathematics. Toward this end, we used measures of white matter microstructure (FA, RD, and $\mathrm{AD}$ ) obtained from quantitative fiber tractography of DTI data to investigate the relation between early brain development and working memory capacity in a large group of healthy infants.

Based on studies of the brain regions and neural circuitry known to support working memory processes during late childhood and adulthood (Courtney et al., 1997; Jonides et al., 1993; Klingberg et al., 2002, 2006; Kwon et al., 2002; Nagy et al., 2004), we hypothesized that the following white matter tracts would be associated with infants' working memory capacity: genu of the corpus callosum, bilateral anterior cingulum, arcuate fasciculi, anterior and superior thalamic radiations, and temporal-parietal segments. To assess the specificity of the structure-function relationships of our hypothesized tracts, we also analyzed the microstructure of control tracts less likely to support the emergence of working memory: the body and splenium of the corpus callosum, inferior longitudinal fasciculi, spinothalamic radiations, and the optic nerves. To determine whether brain-behavior associations were in fact specific to working memory and not a reflection of general intelligence or developmental level, other standard measures of early cognitive development were included in the analyses. To confirm our expectation that working memory is specific to particular 
white matter tracts and not to total white matter or global brain volumes, we examined whether working memory was associated with brain development in general. To our knowledge, this study is among the first demonstrations of brain-behavior associations in infants using DTI and tractography.

\section{Materials and Methods}

\subsection{Participants}

Diffusion weighted imaging (DWI) scans and tests of short-term visuospatial working memory and general cognitive development were acquired in 73 typically developing 12 month-old infants (41 boys and 32 girls). Mean age at assessment was 12 months \pm 21 days. Infant participants included 29 singletons and 44 twins (12 of which were twin pairs). Typically developing infants were selected from two ongoing longitudinal studies: normal controls from a study of early brain development in children at risk for neurodevelopmental disorders (Gilmore et al., 2010a) and a twin study of brain development (Gilmore et al., 2010b). Exclusion criteria were major maternal illness or infection during pregnancy, severe congenital abnormality on fetal ultrasound, and maternal diagnosis of a major psychiatric disorder. Written informed consent was obtained from the parents of all infant participants. The Institutional Review Boards of the University of North Carolina School of Medicine and Duke University Medical Center approved this study. Pregnant women were recruited from the outpatient OB-GYN clinics at these two institutions.

\subsection{Working Memory Assessment}

For the working memory assessment task, infants sitting on their mothers' laps were engaged in 1-2 administrations of a 12-trial hiding game previously described (Reznick, 2009). Administrations were 30 minutes apart. Toys that were determined to be highly attractive to the infant were used. Two hiding wells were used on trials 1-6 and 3 hiding wells were used for trials 7-12. Location of the wells was counterbalanced. Intervals between hiding and encouragement to find the toy were 3, 9, and 15 seconds, and this sequence of delays was repeated 4 times through the 12 possible trials per administration. The same order of location and delay was used for all administrations and all infants. For each trial, the infant watched the toy being placed in a well. All wells were then covered simultaneously, the tray was pulled back from the infant, eye contact was established, and the researcher counted and clapped to continue to draw the infant's gaze away from the wells during the delay. After the delay period infants were prompted to show where the toy was hidden. Working memory scores were calculated as the percentage of searches in the correct location. An administration was included in the analyses if 8 of 12 possible trials were completed. Each infant's best score across the two administrations was used in the analyses. The test-retest reliability of our Working Memory Assessment was determined using the data from 1-year-old infants $(n=83)$ who were tested on two occasions, one week apart, for a different study, but using identical procedures. Infants completed the Working Memory Assessment at two time points, one week apart. The intraclass correlation coefficient (ICC) for 2 fixed raters was .61, which is considered moderate or better, for this age group and the one-week time interval (Columbo et al., 1988; Beck et al., 2011; Bishop et al., 2001; Gnys and Willis, 1991).

\subsection{Additional Measures of Infant Development}

Between administrations of the working memory task, infants were assessed using the Mullen Scales of Early Learning. This standardized assessment includes 5 subscales: visual reception, fine motor, gross motor, expressive language and receptive language (Mullen, 1995). Subscales of the Mullen assessment were combined to create (1) the conventional Mullen Early Learning (cognitive) Composite Score (T-score representing an average of the 
subscales visual reception, fine motor, expressive language and receptive language, (2) a Non-Verbal Developmental Quotient (NVDQ) [(average of the Mullen age equivalent scores for visual reception and fine motor subscales / infant's chronological age) $* 100$ ], and (3) a Verbal Developmental Quotient (VDQ) [(average of the Mullen age equivalent scores for receptive language and expressive language subscales / infant's chronological age) * 100]. Mullen NVQD and VDQ scores have been used by researchers interested in capturing early verbal and nonverbal development separately (Philofsky et al., 2004; Rogers et al., 2003).

\subsection{Image Acquisition}

Magnetic resonance images were acquired on a 3T Siemens scanner (Allegra, Siemens Medical System, Erlangen, Germany). A single shot EPI spin-echo sequence was used to acquire diffusion weighted images with the following parameters $(\mathrm{TR} / \mathrm{TE}=5,200 / 73 \mathrm{~ms}$, slice thickness $=2 \mathrm{~mm}$, in-plane resolution $=2 \times 2 \mathrm{~mm}^{2}$ and 45 slices to cover the whole brain). Seven images were acquired for each slice, 1 without diffusion sensitization $(b=0)$ and 6 with $\mathrm{b}=1,000 \mathrm{sec} / \mathrm{mm}^{2}$ along 6 unique directional diffusion gradients. This sequence was repeated 5 times, resulting in 35 diffusion weighted images (DWI) per scan session. Structural T1-weighted images were obtained using a three-dimensional spoiled gradient $\left(\right.$ FLASH TR $/ \mathrm{TE} /$ Flip Angle $\left.=15 / 7 \mathrm{~ms} / 25^{\circ}\right)$. Proton density and T2-weighted images were obtained with a turbo spin echo (TR / TE1 / TE2 /Flip Angle $=6200 / 20 / 119 \mathrm{~ms} /$ $\left.150^{\circ}\right)$. Spatial resolution was $1 \times 1 \times 1 \mathrm{~mm}$ for T1-weighted images and $1.25 \times 1.25 \times 1.5$ $\mathrm{mm}$ with $0.5 \mathrm{~mm}$ interslice gap for the proton density and T2-weighted images.

\subsection{Diffusion Tensor Imaging Analysis}

Strict DWI/DTI quality control, eddy current, and motion correction were performed (Liu et al., 2010) for all DWI scans using DTIPrep (http://www.nitrc.org/projects/dtiprep). Diffusion-weighted images were up-interpolated to an isotropic $0.55 \mathrm{~mm}$ resolution using windowed sinc interpolation. Diffusion tensors were computed using weighted least squares fitting (Goodlett et al., 2009). Eigenvalues $\left(\lambda_{1} \geq \lambda_{2} \geq \lambda_{3}\right)$ and corresponding eigenvectors were calculated to obtain the diffusion properties, including FA, AD and RD. Skull stripping was performed by applying a binary brain mask to the diffusion property maps. Our fibertract-based DTI processing pipeline included creation of a study-specific DTI atlas, followed by generation of all relevant fiber tracts in this atlas space. We then extracted DTI fiber profiles for all imaging datasets for each participant by sampling the fiber tracts in each atlas-mapped DTI image. These steps are discussed in detail below and also visually represented in Figure 1.

\subsection{DTI Atlas Building}

Individual DTI datasets were deformed into a common coordinate system to enable atlasbased fiber tract analysis. We generated our common coordinate system from all 1-year old study subjects via unbiased DTI atlas building as described in Goodlett et al., 2009. While the common coordinate system was employed to establish corresponding fiber tract locations across all subject scans, the DTI properties were sampled in the original image space using the established transforms to the atlas space. Full-tensor DTI atlases used for this study were created using a two-step process (see Fig. 1). In the first step, we computed an unbiased atlas via diffeomorphic fluid flow registration of intensity-normalized FA images (Joshi et al., 2004; Goodlett et al., 2009; Wang et al., 2011). In the second step, we refined this atlas by remapping all datasets into this unbiased atlas via symmetric logEuclidean demons registration (Wang et al., 2011; Vercauteren et al., 2009). DTI atlases were computed as the average tensor matrix over all mapped tensor images. Two atlases were used to identify and extract the diffusion characteristics of white matter fiber bundles, 
the original atlas created by the two-step methods described above and an arcuate-specific atlas. Creating an arcuate-specific atlas was necessary to mitigate structural variability of this $\mathrm{u}$-shaped fiber bundle that makes this a challenging structure to capture across individuals. Fiber tracing methods, described in the following section, identified the arcuate in 100 individual scans, from our ongoing study of early brain development in children (Gilmore et al., 2010a). Next, a subset of individual scans $(n=28)$ whose left arcuate best coincided with Catani's (2007) description were chosen to compute an arcuate-specific atlas with the same construction methods used for the original atlas, described above. All scans of one-year-old infants in the current study were registered into the arcuate-specific atlas to establish point-to-point correspondence. This method allowed us to overcome complications that often prevent successful deterministic tracking of the arcuate while ensuring sensitivity to individual variations in microstructural characteristics for this tract. As a quality control measure, all DTI datasets were employed and all DTI fibers (with the exception of the arcuate) were tracked on both atlases. Statistical analyses of tracts from the separate atlases showed no difference between the diffusion properties generated or their relation to working memory scores.

\subsection{Fiber Tractography}

Standard streamline DTI tractography (Fillard and Gerig, 2003) was performed on a pediatric atlas built from all 1-year old study subjects (see Fig. 1) using 3D Slicer (version 3.6.3) (http://www.slicer.org). Regions of interest (ROIs) were delineated manually using the Slicer "Editor" on the FA color orientation images (Mori and Zhang, 2006) in all three orthogonal views. A two-ROI approach was employed by generating streamlines with Slicer's "Labelmap Seeding" module from a seed ROI. Streamlines were retained if they also passed through a second target ROI. Fiber-generating seeds were sampled isotropically with 1-mm separation within the two ROIs and streamlines were generated bi-directionally with the ends terminating when the linear measure fell below 0.075. Streamline clustering methods (Gerig et al., 2004) within FiberViewerLight (www.nitric.org/projects/fwlight) were used to remove fiber outliers that greatly deviated from the rest of the bundle. Each fiber bundle consisted of 100-500 DTI fibers.

\subsection{Fiber Descriptions}

Genu-The fibers of the genu correspond with the region of the corpus callosum that primarily contains inter-hemispheric prefrontal connections in the orbitofrontal lobes. (See Fig. 2 for a corresponding FA color map and Fig. 3 for a visualization of the white matter fiber bundles described.) The seeding region was traced on three medial sagittal slices where the center of the genu appears as a bright red bulb on the color map at the anterior end of the corpus callosum. Only fibers ending within the prefrontal lobe were retained.

Anterior cingulum-The cingulum contains the fibers running through the cingulate gyrus. These fibers differ greatly in length, with some running all the way from the anterior temporal gyrus to the orbitofrontal cortex while others make shorter connections between the medial frontal, parietal, and occipital lobes. In the current study, the anterior cingulum was defined as the portion of the cingulum running from the anterior frontal lobe to the parietal lobe. The anterior cingulum is easily seeded from the coronal and axial views in the anterior-posterior-oriented regions (commonly appearing in green in colored FA visualization mode) just above the corpus callosum. Streamlines appearing beyond the parietal lobe were cut.

Thalamic radiations-Thalamic radiations run from the thalamus to the cerebral cortex. The fibers leave the thalamus and pass through the internal capsule along with the corona radiata before radiating outward to the frontal, parietal, and occipital lobes in a fan shape. 
The internal capsule, which is easily distinguished in several axial slices, was used as the initial seeding region for the thalamic radiations. These tracts then separate from the more lateral corona radiata, which descend past the thalamus and into the pons. For our study, we analyzed the anterior thalamic radiations, which run to the prefrontal and anterior frontal cortex as part of the anterior limb of the internal capsule, and the superior thalamic radiations (sensori-motor projections), whose fibers ascend through the posterior limb of the internal capsule as a tight bundle before separating as they pass around the central sulcus and connect to the primary motor and somatosensory cortices.

Arcuate fasciculus-The arcuate fasciculus, as described by Catani et al., 2007, is composed of three divisions. The first is a fiber bundle projecting as an arc from the temporal lobe to the frontoparietal region. It originates in Wernicke's area in the midtemporal lobe, travels superiorly to the level of the corpus callosum, and then turns anteriorly about a vertex and travels to its terminus in the pre- and post-central gyri at the level of the corpus callosum. In our study, this division is referred to as the arcuate fasciculus. The second division is a segment that originates in the same location as the arcuate fasciculus and then projects superiorly to the superior parietal lobe. This is referred to as the temporal-parietal segment in this study. The third division is the Anterior Indirect Segment, not included in our analysis.

Optic nerve-The optic nerve was isolated by identifying the optic chiasm in the midline of the anterior-inferior region of the atlas image in the horizontal slice and then moving anteriorly-inferiorly to this decussation at a point where each optic nerve tract could be clearly visualized. Then in the coronal view at the same vertical level, an ROI inclusive of all anterior-posterior fibers, was drawn over each optic nerve. Deterministic fiber tracking and fiber processing were then accomplished as described previously.

Splenium-The splenium consists of the most posterior fibers in the corpus callosum, which connects the right and left halves of the occipital lobe. The seeding region is the posterior bulb of the corpus callosum on the same three medial sagittal slices used for the genu. Only fibers running to the occipital lobe were retained.

Corpus callosum body-For this study the corpus callosum body refers to the portions of the corpus callosum between the genu and splenium making inter-hemispheric connections in the frontal and parietal lobes. As for the genu and splenium, three medial sagittal slices were used to seed the corpus callosum body and only frontal and parietal lobe connections were retained.

Inferior longitudinal fasciculus-The inferior longitudinal fasciculus is a bundle of long fibers connecting the occipital lobe and the temporal lobe. The fibers appear bright green on the color map (see Fig. 1) as they travel through the central part of the temporal and occipital lobes. A large portion of the occipital lobe was used as the seeding region and only fibers traveling to the anterior portion of the temporal lobe were retained.

Spinothalamic tract-The spinothalamic tract transmits sensory information from the brain stem to the thalamus. The spinal column appears as blue bulbs on the color map (see Fig. 1). Three inferior axial slices were used as the seeding region. Only fibers projecting towards the thalamus were retained. Due to a large number of intersecting fibers just inferior to the thalamus, which lowered the FA, our spinothalamic tract stops before reaching the thalamus. 


\subsection{Profile Generation}

After the bundles had been mapped into the atlas space, the fiber bundles generated from the atlas image were resampled within the DTI images of each subject. FiberViewer was used to define a curvilinear re-parameterization of the bundles by using total length as the parameter (Goodlett et al., 2009). We then extracted DTI property profiles for each subject along each tract for FA, AD, and RD. Each data point represented the DTI average value across the fiber bundle at each specific location. The profile ends were truncated where the group average for FA fell below 0.2 , a value that is often used to indicate the boundary between white and gray matter.

\subsection{Atlas Image Mapping and Quality Control}

Participant DTI images were visually and quantitatively checked for successful mapping into the atlas space. The mapped FA images were first checked for gross deformation errors using MRIWatcher (http://www.ia.unc.edu/dev/download/mriwatcher). Then regional tensors similarity measures for six major white matter regions were evaluated as described by Wang et al., 2011. Finally, tract profile quality control (Wang et al., 2011) was performed via correlational analysis with the atlas tract profile.

\subsection{Statistical Analysis}

For demographic variables, frequency distributions were calculated for categorical variables, and means (standard deviations) were calculated for continuous variables. The $t$-test was used for any between-group differences (male versus female, singleton versus twin) for the cognitive variables and global volumetric measures of the brain. Infants' working memory scores were analyzed as the percentage correct, using the best score from one of two test administrations. Infants' scores appeared normally distributed for all cognitive tests.

Total gray matter volume, total white matter volume, and total tissue volume (calculated as the sum of the total gray matter volume and total white matter volume) were used to measure global brain volume.

The study sample included both singletons and twins, so mixed models regression was used to treat the twins as replicates, while the singletons had no replicates. An unstructured covariance structure was used to capture the correlation between twins in a pair. The association between white matter microstructure and infants' working memory scores was analyzed with a mixed linear regression model, controlling for twin status, gender, gestational age at testing, and additional cognitive measures--NVDQ and VDQ. The mean values for DTI properties (FA, RD, and AD) along each fiber tract were modeled separately, with all tracts included as dependent variables. The formula for our mixed linear regression model was $Y_{i}=\beta_{0}+\beta_{1} X_{1 i}+\beta_{2} X_{2 i}+\beta_{3} X_{3 i}+\beta_{4} X_{4 i}+\beta_{5} X_{5 i}+\epsilon_{i}$. Where $Y_{i}=$ Average DTI Value for subject $i ; \beta_{0}=$ The intercept $i ; X_{1 i}=$ Working Memory for subject $i ; X_{2 i}=$ Verbal Quotient for subject $i ; X_{3 i}=$ Non-Verbal Quotient for subject $i ; X_{4 i}=$ Adjusted Age for subject $i ; X_{5 i}=$ Gender, 1 if gender $=$ male subject for subject $i ; \epsilon_{i}=$ Error for subject $i$.

Examination of all cognitive variables in the mixed model analyses showed there were no outliers, Cook's Distance (values > 1). Scores for all subjects were below the Cook's D criterion value. All statistical hypothesis tests were two-tailed and conducted at a significance level of 0.05. After correcting for multiple comparisons (FDR) alpha levels less than $p=0.026$ should be considered significant, uncorrected $p$-values are reported.

It is problematic in a mixed model to estimate partial correlations because the estimates and test statistics depend on restricted or residual maximum likelihood estimation. Thus, we 
could not estimate partial correlations in these mixed models. We chose an alternate conservative method to estimate effect size by fitting analogous least squares regression analyses, and dropping one twin randomly from each pair, thus removing the dependence that necessitated the use of a mixed model. The reported estimates of partial correlations are conservative because of the lost degrees of freedom due to dropping a twin from each pair. As a result, the actual partial correlations are probably slightly higher than those given.

\section{Results}

\subsection{Participants}

Maternal and infant demographic information for the seventy-three 12-month-old infants included in the study are presented in Table 1. Descriptive statistics for all cognitive assessments and tests of sex and twin status are presented in Table 2. Cognitive summary scores from the Mullen Scales of Early Learning were within the normal range (85-139), confirming that participants were typically developing when the cognitive and imaging measures were collected. No differences were found between males and females or between singletons and twins on any of the cognitive assessments.

\subsection{Working Memory Performance and White Matter Microstructure}

Results highlighted robust associations between infants' working memory performance at 12 months with the diffusion properties FA and RD in each of the white matter tracts hypothesized to support working memory processes. Better working memory scores were significantly associated with higher FA and lower RD in each of the putative working memory tracts. Significant relationships were not observed with AD. In contrast, the only significant relationships found between working memory and the control tracts were for the body of the corpus callosum and the left spinothalamic tract. As expected, working memory scores were positively correlated with FA and negatively correlated with RD and AD. Results are summarized in Table 3, along with partial correlations for the associations between working memory and white matter tracts (FA, RD, and $\mathrm{AD}$ ), controlling for gender, age, twin status, and additional measures of cognition.

\subsection{Relation between Age, Other Cognitive Measures, and White Matter}

Tests of the relationships between white matter and the additional developmental measures (age and the two measures of cognition, the NVDQ and the VDQ, derived from the Mullen Scales of Early Learning) showed surprisingly few associations with white matter tracts especially considering the breadth of associations between working memory performance and white matter. In contrast to working memory, NVDQ and VDQ scores were rarely associated with diffusion properties in selected white matter tracts. Exceptions included significant associations of NVDQ with RD in the bilateral anterior thalamic radiations, and of VDQ with RD in the right superior thalamic radiation and with $\mathrm{AD}$ in the right anterior cingulum and right superior thalamic radiation. See Supplementary Tables 1 and 2 for summary of results and partial correlations between NVDQ, VDQ and diffusion parameters (FA, RD, AD) in the selected white matter tracts.

Similarly, compared to working memory, infants' gestational age at the time of testing was not as strongly associated with diffusion indices in selected white matter tracts and very few associations were found with FA. Significant associations of age were more commonly observed with RD including the genu of the corpus callosum, the anterior thalamic radiations, bilaterally, the left anterior cingulum, the left temporal-parietal segment, and the right superior thalamic radiation. See Table 4 for summary of results and partial correlations between age and and diffusion parameters (FA, RD, $\mathrm{AD}$ ) in the selected white matter tracts. 


\subsection{Tests of Global Brain Volumes}

Working memory scores were not significantly associated with the global measures of brain volume, including total gray matter, total white matter, and total tissue volumes. There were no significant global structural brain differences between singletons $(\mathrm{n}=25)$ and twins $(\mathrm{n}=$ 39) for total gray matter, total white matter, or total tissue volumes. Due to imaging artifacts, structural volume data were not available for 9 of the 73 infants for which DTI data were collected. Additional descriptive data and statistical results are presented in Table 5.

\section{Discussion}

Our results show robust brain-behavior associations in infancy using DTI and a simple assessment of visuospatial working memory. Infants' performance on this task is predictive of widespread white matter microstructure in major association, projection, and callosal fibers that connect frontal, parietal, and temporal regions of the brain. Better working memory scores were associated with higher FA and lower RD values suggesting that infants with better working memory potentially have more advanced white matter maturation, characterized by increased myelination. This interpretation of the findings is consistent with the developmental stage of the infants in the current study, given that the brain is still undergoing rapid, widespread myelination at 12 months of age. Working memory performance associations were not observed with most control tracts or with global measures of brain volume, which suggests greater specificity of the identified associations between working memory and the selected white matter tracts. Interestingly, white matter microstructural characteristics were rarely associated with infants' age or other tests of general and specific cognitive ability. The lack of association between these standard measures of infant physical and cognitive development highlights the utility of this working memory assessment, as a predictor of widespread white matter microstructure in infancy.

Multiple neurobiological factors can affect the diffusion of water molecules across a given tract, including axon compaction and diameter, crossing fibers, and, importantly for this study, myelination (Beaulieu, 2002; Mukherjee and McKinstry, 2006). Because FA is simply an index of directional diffusion, we are primarily interested in the association between RD and working memory, as RD is more proximal to the neuroanatomical processes. RD represents an index of the diffusion of water molecules perpendicular to the fiber bundle, which is thought to provide an index of myelination (Zhang et al., 2009; Song et al., 2003; 2005). While it is unclear which neuroanatomical properties drive changes in $\mathrm{RD}$ across the life span, at 12-months of age, the brain is still undergoing a rapid phase of myelination (Brody et al., 1987; Kinney et al., 1988; Yakovlev and LeCours, 1967), thus we consider myelin content and the myelination process as one plausible explanation for the strong associations found between infants' working memory scores and RD values.

Although we found robust relationships between infants' cognitive function and RD, it is yet to be determined whether early patterns of white matter microstructure are predictive of later myelination or of cognitive function (Fields, 2011). Recent results from our lab (Geng et al., 2012a) however suggest that the health and organization of early white matter connections at one-year of age may determine the structural integrity of white matter connections at twoyears of age. It is well known that the health and integrity of white matter is critical for functions of both the peripheral and central nervous systems. There is increasing evidence showing the integrity of white matter fibers, as measured with DTI, contributes to behavioral and cognitive functioning across the lifespan. Moreover, the use of DTI in developmental and psychiatric research is rapidly extending our knowledge of the white matter characteristics associated with physical abilities and cognitive functions (Catani et al., 2007; Liston et al., 2006; Hu et al., 2011; Karlsgodt et al., 2008; Nagy et al., 2004; Penke et al., 2010). To date, however there are few studies of infant white matter that have used DTI and 
deterministic tractography and even fewer studies that have also investigated the resulting microstructural characteristics in association with infant performance or cognition (Dubois et al., 2008; Niogi et al., 2010; Wolff et al., 2012). If early developmental patterns of white matter microstructure can be shown to predict later structural and functional health of the brain, these early brain-behavior associations will be an important step toward identifying weaknesses in neural connections at a time when the brain is potentially more amenable to intervention. Such findings could lead to the development of targeted intervention strategies to strengthen foundational cognitive capacities, such as working memory, since the formation of other cognitive functions critically depends on working memory (Baddeley, 2003).

Working memory has been considered the 'information processing gate-keeper' and the 'workbench of cognition' and thus plays a privileged role in the development of adaptive cognitive functions (D’Esposito, 2007; Kane and Engle, 2002). Working memory capacity has a pervasive influence over cognitive and social-cognitive competencies, including decision-making, planning, cognitive flexibility, reasoning, and emotion regulation (Goldman-Rakic, 1995; Baddeley, 2003). Moreover, visuospatial working memory capacity has been shown to be more closely related to general fluid intelligence than any of the other early developing cognitive domains (Kane and Engle, 2002). Future studies will be needed to confirm the reported findings and to identify which other white matter connections are important to working memory and how they function as networks and how this changes from infancy to late childhood. Identifying and understanding the putative neural circuitry associated with working memory has implications for understanding typical and atypical cognitive development.

Considering the experience-dependent plasticity of both white matter and working memory demonstrated in school-age children and adults (Klingberg, 2010; Olesen et al., 2003; Scholz et al., 2009; Takeuchi et al., 2010; Zatorre et al., 2012), evidence from the current report suggests intriguing possibilities for identifying developmental periods that are optimal for strengthening children's working memory capacity. Deficits in working memory are a core feature of ADHD and schizophrenia. Implementing working memory training interventions during optimal periods of developmental plasticity could potentially ameliorate cognitive deficits common to these and other neurodevelopmental and neuropsychiatric disorders (Jaeggi et al., 2008; Hagmann et al., 2010; Holtmaat and Svoboda, 2009). Although additional studies are needed to answer fundamental questions about the nature of the interactions between experience-dependent and experience-expectant developmental processes (Johnson, 2001), characterizing the underlying white matter microstructure associated with working memory in infancy provides the groundwork for subsequent longitudinal examinations of these relations.

Results from our mixed regression model, controlling for twin-status, showed an unexpected association between working memory scores and a control tract, the left spinothalamic radiation. However, this result largely disappeared when the sample size was reduced and the methodology changed (i.e., removal twin pairs, $n=12$ ) for the correlational analyses. See Table 3 and section 2.11 in Methods. That this association did not survive the slight perturbation in the dataset and analysis method might suggest that the correlation was spurious to begin with. Although correlations were slightly reduced for the associations between infants' working memory scores and the putative working memory tracts, the general pattern of results and strength of associations remained significant and stable, despite the loss of one twin from each twin pair. The other control tract associated with working memory was the corpus callosum body. This finding may not be surprising given the number of brain regions connected by the body of the corpus callosum which also shares a high degree of overlap with regions known to support working memory that are 
represented with our hypothesized tracts. Our interpretation of this finding is that infants' working memory scores are associated with widespread white matter connections and as such the specificity of these associations will need to be further characterized in future studies.

Consideration should be given to the following study limitations. First, although several white matter tracts were associated with infants' performance, it is likely that additional white matter tracts support working memory in infancy. Thus, future studies of brainbehavior associations should include tract-based spatial statistics to explore brain-wide relationships with white matter (Smith et al., 2006). Second, additional properties of white matter microstructure such as crossing fibers, changes in axonal packing, and even partial volume effects could influence the diffusion of water molecules indexed by FA, RD, and AD (Huppi and Dubois, 2006; Mori and Zhang, 2006; Hermoye, 2006; Mori and van Zijl, 2002; Concha et al., 2010), and this restricts the interpretation of findings. However, a recent study found that autism is associated with abnormalities of white matter development between the ages of 6 and 24 months (Wolff et al., 2012), suggesting that trajectories of white matter microstructural characteristics measured with DTI have the potential to predict complex behavioral patterns. Finally, children who completed at least 8 of 12 working memory trials were included in this study. It is therefore possible that the white matter microstructure differs in those infants who were excluded from the study on the basis of incomplete data, although the working memory performance of infants included in this study ranged widely (from 33-100\% correct) and appeared normally distributed. Factors mediating the current brain-behavior associations are likely to be multiplicative and result from genetic and environmental influences. We did not investigate mediating factors in the current study; future research will be needed to identify mediating factors of white matter maturation and infant learning. More broadly however, the current study does contribute to ongoing efforts to identify early biomarkers and determinants of long-term health and disease.

In summary, using quantitative tractography, this study found robust relationships between infants' working memory and microstructural characteristics of widespread white matter. Significant associations were found for white matter tracts that connect brain regions known to support working memory in older children and adults. Better working memory scores were significantly associated with higher FA and lower RD values in these selected white matter tracts. These strong tract-specific brain-behavior relationships accounted for a significant amount of individual variation above and beyond infants' gestational age and developmental level, as measured with the Mullen Scales of Early Learning.

Future studies will be needed to determine the predictive utility of white matter microstructural characteristics in the infant brain. It is possible that this brain-behavior association resulted from a more rapid developmental trajectory for those infants with better working memory performance and that those infants with poorer performance will catch up over time. Thus, it remains to be seen whether this early brain-behavior association predicts long-term advantages in working memory capacity or other cognitive domains. Our future research will investigate the predictive power of these early brain-behavior relationships as we continue to work with these children that are enrolled in our large longitudinal studies of early brain and behavioral development. Likewise, it will be important to identify genetic and environmental factors that contribute to these individual differences in white matter fiber bundles. We will also address genetic and environmental factors following subsequent assessments of the participants in our large-scale, longitudinal study of brain development in children. 


\section{Supplementary Material}

Refer to Web version on PubMed Central for supplementary material.

\section{Acknowledgments}

This research was supported by the following grants: NIMH Silvio O. Conte Center (MH064065) and Twin (MH070890) and (MH091645), NICHD (HD053000), IDDRC (P30-HD003110), and NRSA (T32 NS007431). SJS and JTE were supported by NRSA awards (T32 HD040127 and 5T32HD007376, respectively) from NICHD. MS and GG were additionally supported by the National Alliance for Medical Image Computing (NA-MIC), funded by the NIH (U54 EB005149). In addition, we gratefully acknowledge Dianne Evans, Mary Norton, Bob Hamer, Amanda Lyall, Xiujuan Geng, and Janice Linn for their contributions to this project, and the research assistants involved in testing the infants: Elizabeth Misiti, Kathryn Cochrane, Hillary Langley, Sarah Palmer, Portia Henderson, Molly McGinnis, and Emily Bostwick. We are also deeply grateful to the families of the infants for their willingness to involve their infants, and themselves, in this work.

\section{References}

Baddeley A. Working memory. Science. 1992; 255:556-559. [PubMed: 1736359]

Baddeley A. Working memory: looking back and looking forward. Nat. Rev. Neurosci. 2003; 4:829_ 839. [PubMed: 14523382]

Beaulieu C. The basis of anisotropic water diffusion in the nervous system-A technical review. NMR Biomed. 2002; 15:435-455. [PubMed: 12489094]

Beck DM, Schaefer C, Pang K, Carlson SM. Executive function in preschool children: Test-retest reliability. J Cogn Dev. 2011; 12(2):169-193. [PubMed: 21643523]

Bengtsson SL, Nagy Z, Skare S, Forsman L, Forssberg H, Ullen F. Extensive piano practicing has regionally specific effects on white matter development. Nat. Neurosci. 2005; 8:1148-1150. [PubMed: 16116456]

Bishop DVM, Aamodt-Leeper G, Creswell C, McGurk R, Skuse DH. Individual differences in cognitive planning on the Tower of Hanoi task: Neuropsychological maturity or measurement error? J Child Psychol Psychiatry. 2001; 42:551-556. [PubMed: 11383971]

Brody BA, Kinney HC, Kloman AS, Gilles FH. Sequence of central nervous system myelination in human infancy. I. An autopsy study of myelination. J Neuropathol Exp Neurol. 1987; 46:283-301. [PubMed: 3559630]

Catani M, Allin MP, Husain M, Pugliese L, Mesulam MM, Murray RM, Jones DK. Symmetries in human brain language pathways correlate with verbal recall. Proc. Natl. Acad. Sci. USA. 2007; 104:17163-17168. [PubMed: 17939998]

Columbo J, Mitchell DW, Horowitz FD. Infant visual attention in the paired-comparison paradigm: Test-retest and attention-performance relations. Child Dev. 1988; 59:1198-1210. [PubMed: 3168636]

Concha L, Livy DJ, Beaulieu C, Wheatley BM, Gross DW. In vivo diffusion tensor imaging and histopathology of the fimbriafornix in temporal lobe epilepsy. J Neurosci. 2010; 30:996-1002. [PubMed: 20089908]

Courtney SM, Ungerleider LG, Keil K, Haxby JV. Transient and sustained activity in a distributed neural system for human working memory. Nature. 1997; 386:608-611. [PubMed: 9121584]

D'Esposito M. From cognitive to neural models of working memory. Philos Trans R Soc Lond B Biol Sci. 2007; 29:761-772. [PubMed: 17400538]

Diamond A. Development of the ability to use recall to guide action, as indicated by infants' performance on A-not-B. Child Dev. 1985; 56:868-883. [PubMed: 4042750]

Diamond, A. Frontal lobe involvement in cognitive changes during the first year of life. In: Gibson, KR.; Petersen, AC., editors. Brain maturation and cognitive development: Comparative and crosscultural perspectives. Aldine de Gruyter; New York: 1991. p. 127-180.

Diamond A, Carlson SM, Beck DM. Preschool children's performance in task switching on the dimensional change card sort task: Separating the dimensions aids the ability to switch. Dev Neuropsych. 2005; 28:689-729. 
Dubois J, Dehaene-Lambertz G, Soarès C, Cointepas Y, Le Bihan D, Hertz-Pannier L. Microstructural correlates of infant functional development: example of the visual pathways. J Neurosci. 2008; 28:1943-1948. [PubMed: 18287510]

Dubois J, Hertz-Pannier L, Cachia A, Mangin JF, Le Bihan D, Dehaene-Lambertz G. Structural asymmetries in the infant language and sensori-motor networks. Cereb Cortex. 2009; 19:414-423. [PubMed: 18562332]

Durston S, Davidson MC, Tottenham N, Galvan A, Spicer J, Fossella JA, Casey BJ. A shift from diffuse to focal cortical activity with development. Dev Sci. 2006; 9:1-8. [PubMed: 16445387]

Elman, JL. Rethinking Innateness: A Connectionist Perspective on Development. MIT Press; Cambridge, MA: 1996.

Fair DA, Dosenbach NUF, Church JA, Cohen AL, Brahmbhatt S, Miezin FM, Barch DM, Raichle ME, Petersen SE, Schlagger BL. Development of distinct control networks through segregation and integration. Proc Natl Acad Sci USA. 2007; 104:13507-13512. [PubMed: 17679691]

Fields RD. Imaging learning: The search for a memory trace. Neuroscientist. 2011; 17:185-196. [PubMed: 21403182]

Fillard P, Gerig G. Analysis tool for diffusion tensor MRI. Medical Image Computing and ComputerAssisted Intervention - MICCAI. 2003; 2879:967-968.

Gao W, Lin W, Chen Y, Gerig G, Smith JK, Jewels V, Gilmore JH. Temporal and spatial development of axonal maturation and myelination of white matter in the developing brain. Am J Neuroradiol. 2009a; 30:290-296. [PubMed: 19001533]

Gao W, Zhu H, Giovanello KS, Smith JK, Shen D, Gilmore JH, Lin W. Evidence on the emergence of the brain's default network from 2-week-old to 2-year-old healthy pediatric subjects. Proc Natl Acad Sci USA. 2009b; 106:6790-6795. [PubMed: 19351894]

Gao W, Gilmore JH, Giovanello K, Smith K, Shen D, Zhu H, Lin L. Temporal and spatial evolution of brain network topology during the first two years of life. PLoS One. 2011; 6:e25278. [PubMed: 21966479]

Geng X, Gouttard S, Sharma A, Gu H, Styner M, Lin W, Gerig G, Gilmore JH. Quantitative tractography of white matter development from birth to age two years. Neuroimage. 2012a (in press).

Geng W, Perez J, Prom-Wormley EC, Kubarych T, Styner M, Lin W, Neale M, Gilmore JH. White matter heritability using diffusion tensor imaging in neonatal brains. Twin Research and Human Genetics. 2012b (in press).

Gerig G, Gouttard S, Corouge I. Analysis of brain white matter via fiber tract modeling. Conf Proc IEEE Eng Med Biol Soc. 2004; 6:4421-4424. [PubMed: 17271286]

Gilmore JH, Kang C, Evans DD, Wolfe HM, Smith KS, Lieberman JA, Lin W, Hamer RM, Styner M, Gerig G. Prenatal and neonatal brain structure and white matter in children at high risk for schizophrenia. Am. J. Psychiatry. 2010a; 167:1083-1091. [PubMed: 20516153]

Gilmore JH, Schmitt JE, Knickmeyer RC, Smith JK, Lin W, Styner M, Gerig G, Neale MC. Genetic and environmental contributions to neonatal brain structure: A twin study. Hum. Brain Mapp. 2010b; 31:1174-1182. [PubMed: 20063301]

Gnys, J.a.; Willis, WG. Validation of executive function tasks with young children. Dev Neuropsychol. 1991; 7:487-501.

Goldman-Rakic PS. Cellular basis of working memory. Neuron. 1995; 14:477-485. [PubMed: 7695894]

Goodlett CB, Fletcher PT, Gilmore JH, Gerig G. Group analysis of DTI fiber tract statistics with application to neurodevelopment. Neuroimage. 2009; 45:S133-142. [PubMed: 19059345]

Gottesman II, Gould TD. The endophenotype concept in psychiatry: etymology and strategic intentions. Am. J. Psychiatry. 2003; 160:636-645. [PubMed: 12668349]

Hagmann P, Sporns O, Madan N, Cammoun L, Pienaar R, Wedeen VJ, Meuli R, Thiran JP, Grant PE. White matter maturation reshapes structural connectivity in the late developing human brain. Proc. Natl. Acad. Sci. USA. 2010; 107:19067-19072. [PubMed: 20956328]

Hermoye L, Saint-Martin C, Cosnard G, Lee S, Kim J, Nassogne MC, Menten R, Clapuyt P, Donohue PK, Hua K, Wakana S, Jiang H, van Zilj PC, Mori S. Pediatric diffusion tensor imaging: normal 
database and observation of the white matter maturation in early childhood. Neuroimage. 2006; 29:493-504. [PubMed: 16194615]

Holtmaat A, Svoboda K. Experience-dependent structural plasticity in the mammalian brain. Nat .Rev. Neurosci. 2009; 10:647-658. [PubMed: 19693029]

Hu Y, Geng F, Tao L, Hu N, Du F, Fu K, Chen F. Enhanced white matter tracts integrity in children with abacus training. Hum. Brain Mapp. 2011; 32:10-21. [PubMed: 20235096]

Huppi PS, Dubois J. Diffusion tensor imaging of brain development. Semin Fetal Neonatal Med. 2006; 11:489-497. [PubMed: 16962837]

Jaeggi SM, Buschkuehl M, Jonides J, Perrig WJ. Improving fluid intelligence with training on working memory. Proc Natl Acad Sci USA. 2008; 105:6829-6833. [PubMed: 18443283]

Johnson MH. Functional brain development in humans. Nat. Rev. Neurosci. 2001; 2:475-483. [PubMed: 11433372]

Jonides J, Smith E.e. Koeppe RA, Awh E, Minoshima S, Mintun MA. Spatial working-memory in humans as revealed by PET. Nature. 1993; 363:623-625. [PubMed: 8510752]

Joshi S, Davis B, Jomier M, Gerig G. Unbiased diffeomorphic atlas construction for computational anatomy. NeuroImage. 2004; 23:S151-160. [PubMed: 15501084]

Kane MJ, Engle RW. The role of the prefrontal cortex in working-memory capacity, executive attention, and general fluid intelligence: an individual-differences perspective. Psych. Bull. Rev. 2002; 9:637-671.

Karlsgodt KH, Van Erp TG, Poldrack RA, Bearden CE, Nuechterlein KH, Cannon TD. Diffusion tensor imaging of the superior longitudinal fasciculus and working memory in recent-onset schizophrenia. Biol. Psychiatry. 2008; 63:512-518. [PubMed: 17720147]

Keller TA, Just MA. Altering cortical connectivity: remediation-induced changes in the white matter of poor readers. Neuron. 2009; 64:624-631. [PubMed: 20005820]

Kinney HC, Brody BA, Kloman AS, Gilles FH. Sequence of central nervous system myelination in human infancy: II. Patterns of myelination in autopsied infants. J. Neuropathol. Exp. Neurol. 1988; 47:217-234. [PubMed: 3367155]

Klingberg T, Forssberg H, Westerberg H. Increased brain activity in frontal and parietal cortex underlies the development of visuospatial working memory capacity during childhood. J Cog Neurosci. 2002; 14:1-10.

Klingberg T. Development of a superior frontal-intraparietal network for visuo-spatial working memory. Neuropsychologia. 2006; 44:2171-2177. [PubMed: 16405923]

Klingberg T. Training and plasticity of working memory. Trends Cogn. Sci. 2010; 14:317-324. [PubMed: 20630350]

Kwon H, Reiss AL, Menon V. Neural basis of protracted developmental changes in visuo-spatial working memory. Proc. Natl. Acad. Sci. USA. 2002; 99:13336-13341. [PubMed: 12244209]

Lebel C, Walker L, Leemans A, Phillips L, Beaulieu C. Microstructural maturation of the human brain from childhood to adulthood. NeuroImage. 2008; 40:1044-1055. [PubMed: 18295509]

Liston C, Watts R, Tottenham N, Davidson MC, Niogi S, Ulug AM, Casey BJ. Frontostriatal microstructure modulates efficient recruitment of cognitive control. Cereb. Cortex. 2006; 16:553560. [PubMed: 16033925]

Liu Z, Wang Y, Gerig G, Gouttard S, Tao R, Fletcher T, Styner M. Quality control of diffusion weighted images. Proceedings of SPIE. 2010; 7628:76280J.

Markham JA, Greenough WT. Experience-driven brain plasticity: beyond the synapse. Neuron Glia Biol. 2004; 1:351-363. [PubMed: 16921405]

Mori S, Zhang J. Principles of diffusion tensor imaging and its applications to basic neuroscience research. Neuron. 2006; 51:527-539. [PubMed: 16950152]

Mori S, van Zijl PC. Fiber tracking: principles and strategies - a technical review. NMR Biomed. 2002; 15:468-480. [PubMed: 12489096]

Mukherjee P, McKinstry RC. Diffusion tensor imaging and tractography of human brain development. Neuroimag Clin N Am. 2006; 16:19-43. 
Mukherjee P, Miller JH, Shimony JS, Conturo TE, Lee BC, Almli CR, McKinstry RC. Normal brain maturation during childhood: developmental trends characterized with diffusion tensor imaging. Radiology. 2001; 221:349-58. 2001. [PubMed: 11687675]

Mullen, EM. Mullen Scales of Early Learning: AGS Edition. AGS Publishing; Circle Pines, Minn: 1995.

Nagy Z, Westerberg H, Klingberg T. Maturation of white matter is associated with the development of cognitive functions during childhood. J. Cogn. Neurosci. 2004; 16:1227-1233. [PubMed: 15453975]

Niogi S, Mukherjee P, Ghajar J, McCandliss B. Individual differences in distinct components of attention are linked to anatomical variation in distinct white matter tracts. Front Neuroanat. 2010; 4:1-12. [PubMed: 20161990]

Olesen PJ, Westerberg H, Klingberg T. Increased prefrontal and parietal brain activity after training of working memory. Nat. Neurosci. 2003; 7:75-79. [PubMed: 14699419]

Paus T, Collins DL, Evans AC, Leonard G, Pike B, Zijdenbos A. Maturation of white matter in the human brain: a review of magnetic resonance studies. Brain Res. Bull. 2001; 54:255-266. [PubMed: 11287130]

Pelphrey KA, Reznick JS, Davis Goldman B, Sasson N, Morrow J, Donahoe A, Hodgson K. Development of visuospatial short-term memory in the second half of the $1^{\text {st }}$ year. Dev. Psychol. 2004; 40:836-851. [PubMed: 15355170]

Penke L, Munoz Maniega S, Murray C, Gow AJ, Hernandez MC, Clayden JD, Starr JM, Wardlaw JM, Bastin ME, Deary IJ. A general factor of brain white matter integrity predicts information processing speed in healthy older people. J. Neurosci. 2010; 30:7569-7574. [PubMed: 20519531]

Philofsky A, Hepburn SL, Hayes A, Hagerman R, Rogers SJ, Abbeduto L. Linguistic and cognitive functioning and autism symptoms in young children with fragile $\times$ syndrome. Am J Ment Retard. 2004; 109:208-218. [PubMed: 15072521]

Quartz SR, Sejnowski TJ. The neural basis of cognitive development: a constructivist manifesto. Behav. Brain Sci. 1997; 20:537-596. [PubMed: 10097006]

Reznick JS, Morrow JD, Goldman BD, Snyder J. The onset of working memory in infants. Infancy. $2004 ; 6: 145-154$.

Reznick, JS. Working memory in infants and toddlers. In: Courage, ML.; Cowan, N., editors. The Development of Memory in Infancy and Childhood. Psychology Press; New York, NY: 2009. p. 343-365.

Rogers SJ, Hepburn SL, Stackhouse T, Wehner E. Imitation performance in toddlers with autism and those with other developmental disorders. J Child Psychol Psychiatry. 2003; 44:763-781. [PubMed: 12831120]

Scherf KS, Sewwney JA, Luna B. Brain basis of developmental change in visuospatial working memory. J Cogn Neurosci. 2006; 18:1045-1058. [PubMed: 16839280]

Schmithorst VJ, Wilke M, Dardzinski BJ, Holland SK. Correlation of white matter diffusivity and anisotropy with age during childhood and adolescence: a cross-sectional diffusion-tensor MR imaging study. Radiology. 2002; 222:212-218. [PubMed: 11756728]

Schmithorst VJ, Wilke M, Dardzinski BJ, Holland SK. Cognitive functions correlate with white matter architecture in normal pediatric population: a diffusion tensor MRI study. Hum Brain Mapp. 2005; 26:139-47. [PubMed: 15858815]

Scholz J, Klein MC, Behrens TEJ, Johansen-Berg H. Training induces changes in white-matter architecture. Nat. Neurosci. 2009; 12:1370-1371. [PubMed: 19820707]

Smith SM, Jenkinson M, Johansen-Berg H, Rueckert D, Nichols TE, Mackay CE, Watkins KE, Ciccarelli O, Cader MZ, Matthews PM, Behrens TEJ. Tract-based spatial statistics: voxelwise analysis of multi-subject diffusion data. NeuroImage. 2006; 31:1487-1505. [PubMed: 16624579]

Song SK, Sun SW, Ju WK, Lin S,J, Cross AH, Neufeld AH. Diffusion tensor imaging detects and differentiates axon and myelin degeneration in mouse optic nerve after retinal ischemia. NeuroImage. 2003; 20:1714-1722. [PubMed: 14642481]

Song SK, Yoshino J, Le TQ, Lin SJ, Sun SW, Cross AH, Armstrong RC. Demyelination increases radial diffusivity in corpus callosum of mouse brain. Neuroimage. 2005; 25:132-140. [PubMed: $15862213]$ 
Takeuchi H, Sekiguchi A, Tai Y, Yokoyama S, Yomogida Y, et al. Training of working memory impacts structural connectivity. J Neurosci. 2010; 30:3297-3303. [PubMed: 20203189]

Thomas B, Eyssen M, Peeters R, Molenaers G, Van Hecke P, De Cock P, Sunaert S. Quantitative diffusion tensor imaging in cerebral palsy due to periventricular white matter injury. Brain. 2005; 128:2562-2577. [PubMed: 16049045]

Uddin LQ, Supekar KS, Ryali S, Menon V. Dynamic reconfiguration of structural and functional connectivity across core neurocognitive brain networks with development. J. Neurosci. 2011; 31:18578-18589. [PubMed: 22171056]

Vercauteren T, Pennec X, Perchant A, Ayache N. Diffeomorphic demons: efficient non-parametric image registration. Neuroimage. 2009; 45:S61-72. [PubMed: 19041946]

Wang Y, Gupta A, Liu Z, Zhang H, Escolar ML, Gilmore JH, Gouttard S, Fillard P, Maltbie E, Gerig G, Styner M. DTI registration in atlas based fiber analysis of infantile Krabbe disease. Neuroimage. 2011; 55:1577-1586. [PubMed: 21256236]

Werring DJ, Clark CA, Barker GJ, Thompson AJ, Miller DH. Diffusion tensor imaging of lesions and normal-appearing white matter in multiple sclerosis. Neurology. 1999; 52:1626-1632. [PubMed: 10331689]

Wolff JJ, Gu H, Gerig G, Elison JT, Styner M, Gouttard S, Botteron KN, Dager SR, Estes AM, Hazlett HC, Kostopoulos P, McKinstry RC, Paterson SJ, Schultz RT, Zwaigenbaum L, Piven J. Differences in white matter fiber tract development present from 6 to 24 months in infants with autism. Am J Psychiatry. 2012 (in press).

Yakovlev, PI.; Lecours, AR. The myelogenetic cycles of regional maturation of the brain. In: Minkowski, A., editor. Regional development of the brain in early life. Blackwell Scientific Publications; Oxford: 1967. p. 3-65.

Zald DH, Iacono WG. The development of spatial working memory abilities. Dev Neuropsychol. 1998; 14:563-578.

Zatorre RJ, Fields RD, Johansen-Berg H. Plasticity in gray and white: neuroimaging changes in brain structures during learning. Nat Neurosci. 2012; 15:528-536. [PubMed: 22426254]

Zelazo, PD.; Carlson, SM.; Kesek, A. Development of executive function in childhood. In: Nelson, CA.; Luciana, M., editors. Handbook of developmental cognitive neuroscience. 2nd ed. MIT Press; Cambridge, MA: 2008. p. 553-574.

Zelazo PD, Frye D, Rapus T. An age-related dissociation between knowing rules and using them. Cogn Dev. 1996; 11:37-63.

Zhang J, Jones M, DeBoy CA, Reich DS, Farrell JA, Hoffman PN, Griffin JW, Sheikh KA, Miller MI, Mori S, Calabresi PA. Diffusion tensor magnetic resonance imaging of Wallerian degeneration in rat spinal cord after dorsal root axotomy. J. Neurosci. 2009; 29:3160-3171. [PubMed: 19279253] 


\section{Highlights}

Working memory emerges early in life and is critical for other cognitive functions. Infants' working memory is robustly associated with white matter microstructure.

Tracts connecting brain regions known to support working memory were associated.

Infants' age and general cognition were not as strongly associated with white matter.

These results improve our understanding of brain-behavior relationships in infancy. 


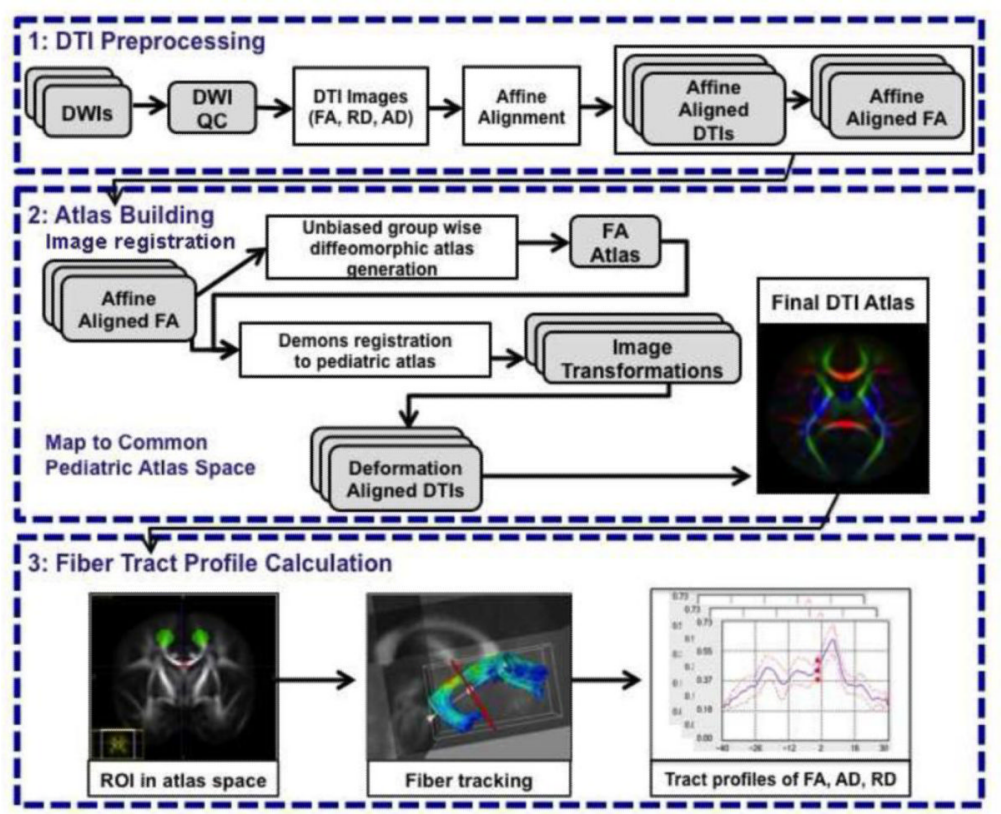

Figure 1.

Flow Chart of the DTI Fiber Tract-based Analysis. The first step, DTI preprocessing includes quality control of the tensor estimation and computation of DTI indices: FA, RD and $\mathrm{AD}$. The second step, atlas construction, includes image registration and mapping DTIs to common space: a) construction of an unbiased pediatric FA atlas using group-wise diffeomorphic registration, $b$ ) mapping all datasets into the pediatric FA atlas with Demons registration, and c) mapping DTIs to common space and averaging the transformed DTIs to get the final pediatric DTI atlas. The third and final step, fiber tract profile calculation, includes standard fiber tractography in atlas space, followed by DTI indices profile extraction along the fiber tracts using the atlas transformation computed in step two. 


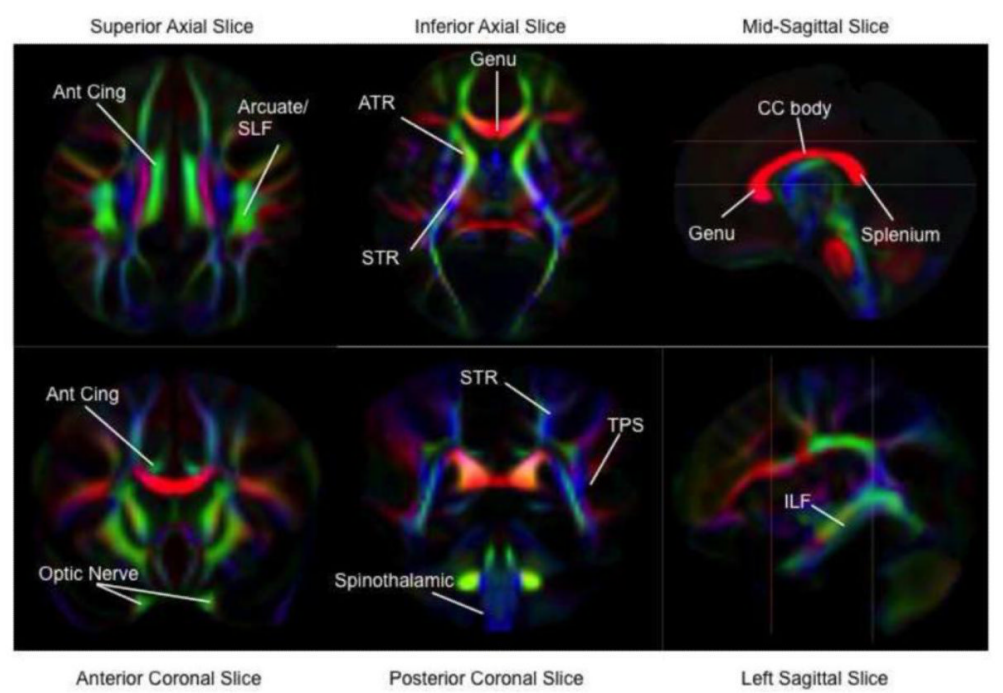

Figure 2.

DTI Fractional Anisotropy (FA) Maps of White Matter Tracts. FA maps are color-coded by local fiber direction. The colors, red, green, and blue represent white matter fibers running along the right-left, anterior-posterior, and superior-inferior axes, respectively. Locations of white matter tracts are assigned on the color maps. Reference lines in sagittal images indicate respective locations of axial and coronal images. Temporal-parietal segment (TPS), inferior longitudinal fasiculus (ILF), body of the corpus callosum (CC Body), anterior cingulum (Ant. Cing), anterior thalamic radiations (ATR) part of the anterior limb of the internal capsule, superior thalamic radiations (STR) part of the posterior limb of the internal capsule. 


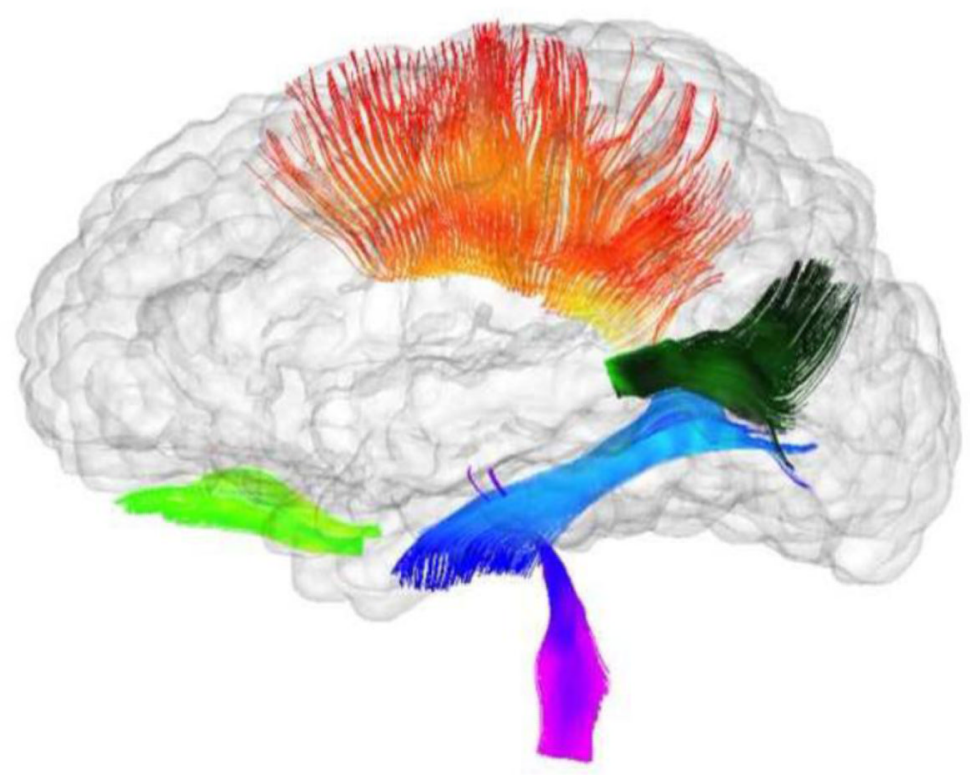

A

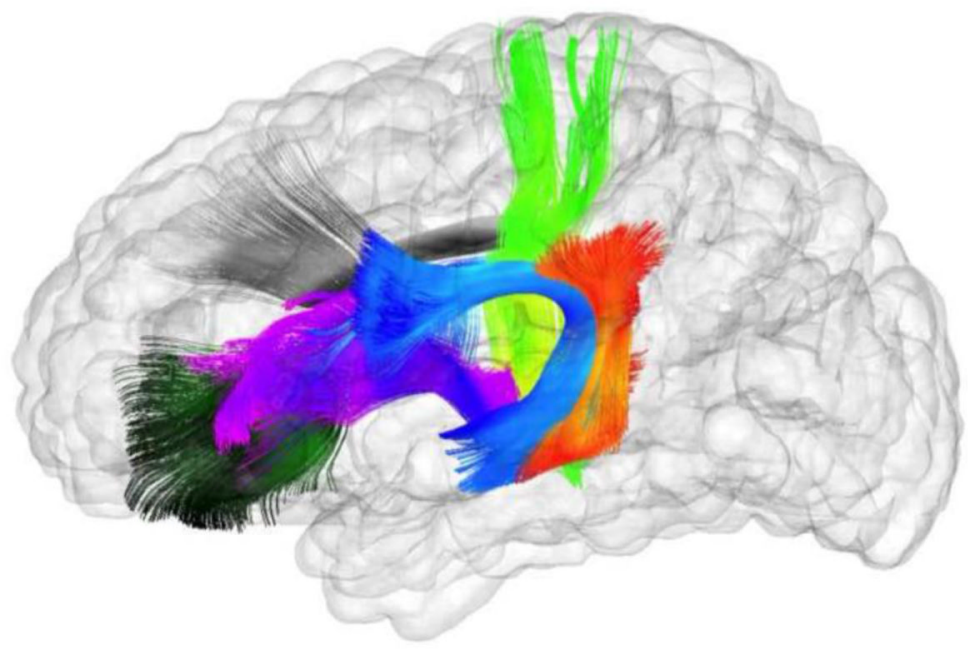

B

Figure 3.

White Matter Fiber Bundles from Tractography of DTI Data. This figure contains two glass brain images showing the reconstructed fibers included in this study-- a. Working Memory Tracts: anterior cingulum (dark blue), superior thalamic radiations (bright green), genu (dark green), temporal-parietal segment (orange), anterior thalamic radiations (purple), arcuate fasciculus (light blue). b. Control Tracts: optic nerves (bright green), corpus callosum body (orange), splenium (dark green), inferior longitudinal fasciculi (blue), spinothalamic radiations (purple). Standard streamline DTI tractography was performed on a pediatric atlas built from all 1-year old study subjects. 

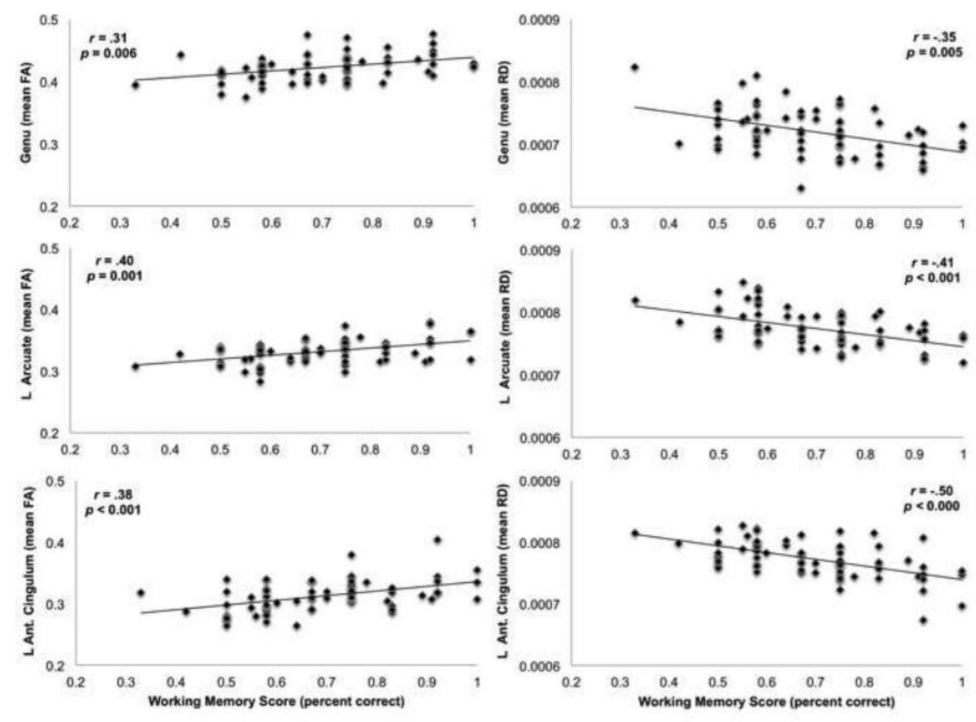

Figure 4.

Association Between Working Memory and White Matter Tracts. Scatter plots highlight examples of significant associations between infants' working memory and key white matter tracts: Genu, Arcuate, Anterior Cingulum. Plots show the positive association of working memory with FA and the negative association with RD. Only the Left (L) Arcuate and L Ant. Cingulum are shown above, however scatter plots for these tracts in the right hemisphere are very similar. Partial correlations and uncorrected $p$-values highlight the magnitude of these associations. 


\section{Table 1}

Infant and Maternal Demographics

\begin{tabular}{lc}
\hline Birth Weight (grams) & $2767 \pm 804$ \\
Gestational Age at Birth (weeks) & $37.1 \pm 3$ \\
Age at Assessment/MRI (months) & $12 \pm .7$ \\
Maternal Age at Birth (years) & $29 \pm 6$ \\
Maternal Education Level (years) & $14 \pm 4$ \\
Ethnicity of Mother (n) & \\
Caucasian & 35 \\
African American & 27 \\
Hispanic/Latino & 11 \\
\hline
\end{tabular}

Demographic information based on the entire sample $(n=73)$. Values represent Mean \pm SD 
Table 2

Descriptive Statistics for Cognitive Data and Tests of Gender and Twin Status

\begin{tabular}{|c|c|c|c|c|c|c|c|}
\hline \multirow{2}{*}{$\begin{array}{l}\text { Infant Cognitive } \\
\text { Assessments }\end{array}$} & $\begin{array}{r}\text { Males } \\
(\mathrm{n}=\mathbf{4 1})\end{array}$ & $\begin{array}{r}\text { Females } \\
(\mathbf{n}=\mathbf{3 2})\end{array}$ & \multirow[b]{2}{*}{$p$-value } & $\begin{array}{r}\text { Singletons } \\
(\mathbf{n}=\mathbf{2 9})\end{array}$ & $\begin{array}{r}\text { Twins } \\
(\mathbf{n}=\mathbf{4 4})\end{array}$ & \multirow[b]{2}{*}{$p$-value } & $\begin{array}{r}\text { Total Sample } \\
(\mathbf{N}=\mathbf{7 3})\end{array}$ \\
\hline & \multicolumn{2}{|c|}{ Mean (SD) } & & \multicolumn{2}{|c|}{ Mean (SD) } & & Mean (SD) \\
\hline Working Memory & $69(16)$ & $70(14)$ & 0.62 & $70(14)$ & $68(16)$ & 0.62 & $69(15)$ \\
\hline Mullen ELCS & $115(13)$ & $113(13)$ & 0.52 & $114(13)$ & $114(13)$ & 0.70 & $114(13)$ \\
\hline NVDQ & $122(13)$ & $120(13)$ & 0.54 & $121(10)$ & $121(14)$ & 0.89 & $121(13)$ \\
\hline VDQ & $107(13)$ & $104(12)$ & 0.38 & $106(13)$ & $106(12)$ & 0.86 & $105(13)$ \\
\hline
\end{tabular}

Results from $t$-tests of gender and twin status are shown for all cognitive assessments. Working memory scores reflect the percent correct using the infants' best performance between two test administrations. Mullen Early Learning Composite Score (ELCS) is a standardized score (mean $=100$, $\mathrm{SD} \pm 15$ ). Non-Verbal and Verbal Developmental Quotients (NVDQ, VDQ, respectively) are derived from combining subscales on the Mullen assessment. 


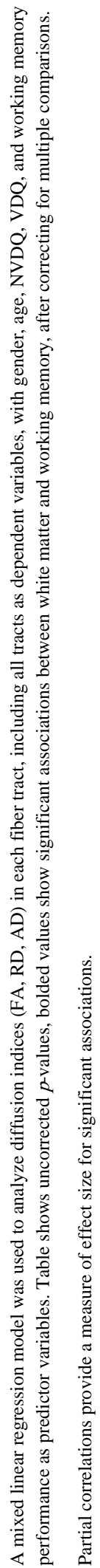




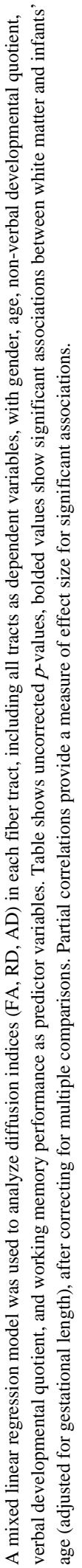


Table 5

Brain Volume Descriptive Statistics for Gender and Twin Status Comparisons

\begin{tabular}{lccc}
\hline Gender & $\begin{array}{c}\text { Male } \\
(\mathrm{n}=34)\end{array}$ & $\begin{array}{c}\text { Female } \\
(\mathrm{n}=30)\end{array}$ & $p$-value \\
\hline Total Tissue Volume & $876,834 \pm 73,015$ & $819,059 \pm 81,067$ & 0.004 \\
Grey Matter & $639,501 \pm 50,847$ & $604,593 \pm 54,091$ & 0.010 \\
White Matter & $237,333 \pm 29,156$ & $214,466 \pm 28,142$ & 0.002 \\
\hline Twin Status & $\begin{array}{c}\text { Singleton } \\
(\mathrm{n}=25)\end{array}$ & $\begin{array}{c}\text { Twin } \\
(\mathrm{n}=39)\end{array}$ & $p$-value \\
\hline Total Tissue Volume & $842,941 \pm 77,054$ & $860,378 \pm 88,850$ & 0.409 \\
Grey Matter & $618,132 \pm 51,438$ & $630,947 \pm 60,051$ & 0.366 \\
White Matter & $224,809 \pm 28,222$ & $229,431 \pm 34,618$ & 0.561 \\
\hline
\end{tabular}

Values are Mean $\pm \mathrm{SD}\left(\mathrm{mm}^{3}\right)$. Structural volumetric data was not available for all infants with DTI. 